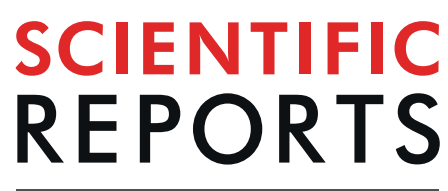

natureresearch

\title{
Knowledge status and sampling strategies to maximize cost- benefit ratio of studies in landscape genomics of wild plants
}

\begin{abstract}
Alesandro Souza Santos* \& Fernanda Amato Gaiotto
To avoid local extinction due to the changes in their natural ecosystems, introduced by anthropogenic activities, species undergo local adaptation. Landscape genomics approach, through genomeenvironment association studies, has helped evaluate the local adaptation in natural populations. Landscape genomics, is still a developing discipline, requiring refinement of guidelines in sampling design, especially for studies conducted in the backdrop of stark socioeconomic realities of the rainforest ecologies, which are global biodiversity hotspots. In this study we aimed to devise strategies to improve the cost-benefit ratio of landscape genomics studies by surveying sampling designs and genome sequencing strategies used in existing studies. We conducted meta-analyses to evaluate the importance of sampling designs, in terms of (i) number of populations sampled, (ii) number of individuals sampled per population, (iii) total number of individuals sampled, and (iv) number of SNPs used in different studies, in discerning the molecular mechanisms underlying local adaptation of wild plant species. Using the linear mixed effects model, we demonstrated that the total number of individuals sampled and the number of SNPs used, significantly influenced the detection of loci underlying the local adaptation. Thus, based on our findings, in order to optimize the cost-benefit ratio of landscape genomics studies, we suggest focusing on increasing the total number of individuals sampled and using a targeted (e.g. sequencing capture) Pool-Seq approach and/or a random (e.g. RADSeq) Pool-Seq approach to detect SNPs and identify SNPs under selection for a given environmental cline. We also found that the existing molecular evidences are inadequate in predicting the local adaptations to climate change in tropical forest ecosystems.
\end{abstract}

Anthropogenic activities are transforming natural systems, drastically changing the environmental conditions in a way which poses major threat to global biodiversity ${ }^{1,2}$. Anthropogenic modifications can lead to reduction and fragmentation of natural environments, or to changes in climatic conditions ${ }^{3-5}$. Species respond to such changes in the natural habitat, by (i) phenotypic plasticity, (ii) migrating from their natural habitats, in search of conditions, fit for survival and having ample resources, or (iii) adapting to the new environment to avoid local extinction (local adaptation $)^{4,6-8}$.

A species is said to exhibit local adaptation, when individuals on an average have a superior fitness in their home environment, compared to a transplanted individual ${ }^{8-11}$. Therefore, local adaptation is driven by the action of natural selection, which acts on the individual's phenotypes, determining the characteristics that will be favored under certain environmental conditions ${ }^{9,12,13}$. Historically, local adaptation has been studied either through translocation experiments (between environments) or trials in the greenhouse under controlled environmental conditions ${ }^{8,14,15}$. The drawbacks of these two approaches include the requirement of ample financial resources and time, which is generally scarce for studies involving long-lived species such as trees ${ }^{14,15}$. Landscape genomics, a study that identifies genetic variations that confer local adaptation, is used to remedy these limitations ${ }^{16}$. In this approach, significant differences in allele frequency between populations of the target species, indicates that individuals in the population are experiencing selection pressure; possibly in response to change in some environmental factor, such as changes in soil type, radiation, water stress, and temperature ${ }^{17-20}$. Landscape genomics studies analyze frequency distribution changes in molecular markers, such as single nucleotide polymorphism 
$(\mathrm{SNPs})^{14,21}$ in relation to given environmental factors. The SNPs are commonly used in wild local adaptation studies because their location and functional annotations are known and they are widely distributed throughout the genome $^{22,23}$. Additionally, the advent of high-throughput sequencing technology (HTS) has made the sequencing of millions of SNPs from the genome, possible, at moderate cost and it is not time intensive ${ }^{24,25}$. Thus, through the use of landscape genomics, it is now possible to find correlations between genomic regions and the variable environmental characteristics ${ }^{15,21}$. Therefore, this approach is used to pinpoint the environmental change in nature, that affects ecology (e.g.: climatic changes) of a species and can influence its adaptive genetic potential ${ }^{26-28}$. Thus, landscape genomics approach is useful in predicting the responses of species to environmental heterogeneity and variable landscape factors ${ }^{29}$.

In landscape genomics studies, outlier loci method has been used in tandem with genome-environment association (GEA) method, to evaluate local adaptation in natural populations ${ }^{21}$. In the outlier loci method, changes in among-population frequency distribution of given allele(s)/loci, significantly different from that seen in the absence of natural selection, are identified, and these alleles/loci are considered to be under natural selection pressure $^{15,21}$. While, in the GEA analysis, occurrence of high correlation between the allele frequencies with one or more environmental variables is considered an indicator of local adaptation ${ }^{10,30}$. However, as the outlier method does not specify the environmental forces at work on the locus under selection, studies using landscape genomics approach use the GEA results ${ }^{31,32}$ to fix the causative environmental factor. For this reason, in this study we tried to systematize and discuss the results obtained from GEA in wild populations, sampled in situ.

Considering that landscape genomics is a relatively new discipline, it requires refinement within the scope of sampling design, for GEA studies ${ }^{33-37}$. Studies, based on simulations, have predicted that the number of SNP markers used and the number of populations sampled influence the inferences from landscape genomics analy$\operatorname{sis}^{38,39}$. However, a recent review article, evaluated the impacts of sampling design on inferences from empirical studies on landscape genomics and it does not take into account the particularities of tropical regions ${ }^{37}$. In a way, our approach complements that of Ahrens et al., as they focused on the limitations of the different techniques, the lack of standardization and non-availability of information in conducting these studies. However, unlike others, our study, by reporting on empirically observed patterns in landscape genomics, aims to shape sampling design strategies for improving the cost-benefit ratio of future works, conducted in laboratories using population genomics for the first time. Guidelines put forth in this study will be helpful, in particular, in subsidizing the costs of studies being carried out in the stark socioeconomic realities of tropical regions, which are global biodiversity hotspots under grave anthropogenic threat.

\section{Methodology}

To perform this work, all research articles, published to date (at the time of writing, September 2018), which evaluated local adaptations in populations of wild plants were pooled. The papers were analyzed for (i) number of populations sampled, (ii) number of individuals sampled per population, (iii) total number of individuals sampled, and (iv) number of SNPs used. Care was taken to ensure that all data were obtained in empirical studies assessing local adaptation in wild plant populations. The Scopus (https://www.scopus.com) and Google Scholar (http://scholar.google.com.br/) databases were searched for title, abstract, and articles using the following keywords: environmental variables and SNPs, landscape genetics and SNPs, spatial analysis and SNPs, landscape genomics and SNPs, population genomics and SNPs, adaptive genetic variation, and local adaptation and SNPs. Search results were filtered to remove papers and reviews from clinical, biomedical, veterinary, and immunology areas. Then, a second filter was applied to eliminate the articles containing the following words: animal, fish, ecology of freshwater, marine biology, entomology, and zoology. Finally, papers using simulations, or involving exotic and crop species in plantations, or using greenhouse experiments were removed and the remaining 35 empirical studies on in situ wild plants were selected for the present work. Using this data set and ArcGIS (10.2), a map was created depicting the distribution of the localities where researches were performed, in the different terrestrial biomes (Fig. 1). A double check was done at this stage to ensure if all the 35 papers chosen for this study, had evaluated in situ local adaptation in wild plant populations, using SNPs markers. The following information from the papers: (1) authors names; (2) publication year, (3) country of study and geographical coordinates; (4) botanical family studied; (5) species; (6) total number of individuals; (7) number of populations; (8) number of SNPs used; (9) number of SNPs under selection; and (10) method used to generate the SNPs data, was compiled.

For this study, we considered as SNPs under selection, the SNPs from the pooled data that had a significant association with a geoclimatic variable, such as temperature, precipitation, latitude, longitude, elevation, evapotranspiration, and drought. They were used as a proxy to detect the potential for natural selection in wild plant populations. The number of SNPs under selection could be influenced by the methods employed to analyze the correlation between allelic frequency and environmental variables ${ }^{38}$, therefore most articles in the literature make inferences from such studies, using conservative criteria ${ }^{34,35,40}$. It is known that each method has different types of limitations and caveats (e.g., vary in the rates of false positives or of false negatives), which can cause errors in the estimates and consequently in the estimate of the number of SNPs under selection ${ }^{38}$. For this reason, we chose to be conservative, using only the results obtained either simultaneously by at least two methods of analysis or strictly controlled for rates of false positives or false negatives (e.g., studies that controlled the population structure in the analyses). Thus, although limitations of the different methodologies used were not fully circumvented, we believe that the results reported in our mini-review, portrays the findings of the literature, in a way seeking to minimize, as much as possible, the biases inherent to the different analysis.

The methods used to generate the SNPs data were subdivided into Random, Random Pool-Seq, Targeted, and Targeted-Pool-Seq categories. The Random category included studies that used random regions of DNA and individualized sequencing for the preparation of libraries. The Random-Pool-Seq category is distinct from Random, in that the data is sequenced with the Pool-Seq technique (Pool-Seq, an equimolar amount of DNA is taken from each individual from a population and pooled for sequencing). In the Targeted category, we included studies that 


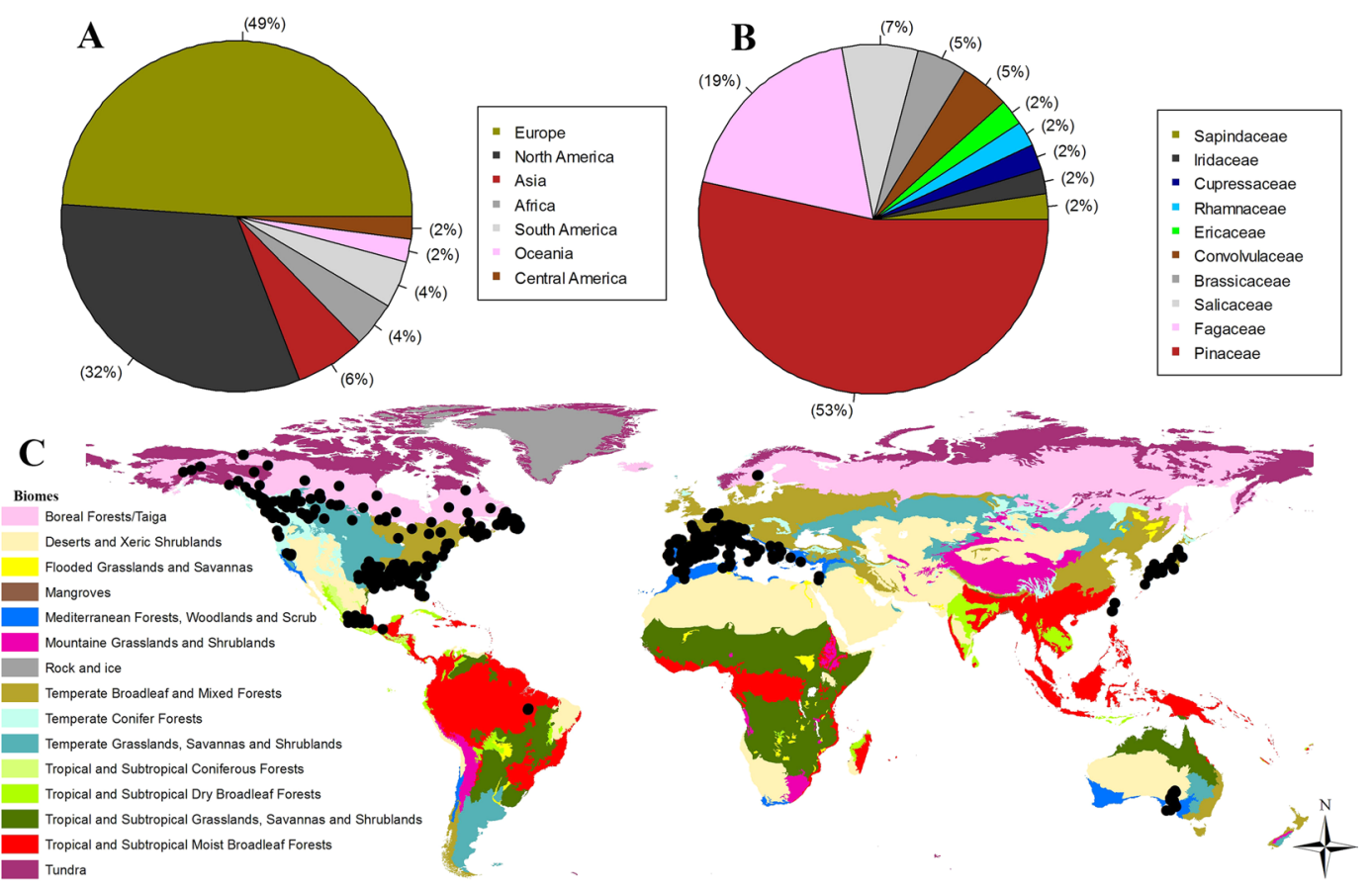

Figure 1. Geographical, ecological and species distribution found in the 35 research articles, included in this study, which evaluated local adaptation in wild plant populations in their natural occurrence area using SNPs markers, are shown; (A) Percentage of studies published per continent; (B) Percentage of studies distributed per plant family; (C) The points distributed on the map indicating the sampling of the studies in the different terrestrial biomes.

used only specific gene regions or expressed sequence tags with individualized sequencing. The Targeted Pool-Seq category differed from Targeted, such that the data is sequenced by Pool-Seq technique mentioned above.

Influence of sample size on number of SNPs under selection was inferred by determining the average of the individual number of samples from each population which was arrived at by dividing the total number of individuals and the number of populations in each study. Additionally, the percentage of SNPs under selection was calculated to estimate the number of genes under natural selection in the genome. Then, a descriptive statistical analysis (minimum, maximum, mean, and standard) was performed with all the variables in R program (http:// www.r-project.org/).

To evaluate the influence of each variable on the number of SNPs under selection, the linear mixed effects model of the lme4 package of $\mathrm{R}$ program ${ }^{41}$ was used. The variables in this study, such as the number of populations, number of individuals, average number of individuals sampled per population, and number of SNPs used were investigated to determine their influence on the number of SNPs under selection and, consequently, the detection of natural selection in plant species. As this type of model requires the fulfillment of the assumptions of normality and homoscedasticity of the residues, we performed the scaling of each variable separately. We used the rank function in R program to reduce the amplitude of our data, meet the assumptions, and obtain the goodness of fit of the statistical models. For each variable, an independent model was created by considering each variable as the fixed effect and the others as the random effect, such that:

$$
\begin{aligned}
& \mathrm{n}^{\mathrm{o}} \text { of SNPs under selection } \sim \mathrm{n}^{\mathrm{o}} \text { of SNPs used }+\left(1 \mid \mathrm{n}^{\mathrm{o}} \text { of individuals }\right) \\
& +\left(1 \mid \mathrm{n}^{\mathrm{o}} \text { of pop }\right)+\left(1 \mid \mathrm{n}^{\mathrm{o}} \text { of ind_ pop }\right) .
\end{aligned}
$$

where, the number of SNPs under selection is the response variable; number of SNPs used is the fixed-effect variable; the terms in parentheses are the random effect variables and the number 1 indicates that the intercept is random between the observations of each variable.

The linear mixed effects model in the lme4 package does not provide the results as the coefficient of determination $\left(r^{2}\right)$, due to theoretical problems or difficulty of implementation. For this reason, we used the marginal $\mathrm{r}^{2}$ to calculate the amount of variance explained by the fixed-effect variables in each model ${ }^{42}$. Finally, we used the function $\mathrm{lm}$ in $\mathrm{R}$ to establish the simple linear regression analysis of the number of SNPs used in the function of the total number of individuals. To make the graphs with the significant results of all the analyses, we used the ggplot2 package in $\mathrm{R}^{43}$. 


\section{Results}

Based on the 35 selected empirical articles, we analyzed 44 observations involving 36 different species for the number of SNPs under selection (Table 1). These studies were conducted mainly in Europe and North America (Fig. 1A), in ten plant families, dominance by Pinaceae (53\% of the species) (Fig. 1B), distributed mainly in biomes of temperate broadleaf and mixed forest, temperate grasslands and Mediterranean forests (Fig. 1C). The total number of individuals ranged from 22 to 2,574, and the number of SNPs used varied between 33 and $2,091,957$. However, the number of SNPs under selection (SNPs with significant association with some geoclimatic variable) varied from 2 to 2,522 with the percentage of SNPs under selection ranging from 0.02 to 78.14 (Table 1).

In the analysis using the linear mixed-effects models, we verified the effect of each variable (related to sample size and SNP number) individually on the number of SNPs under selection, whereas the others were used as random effect, as mentioned in the methodology section. On entering, the number of populations, as fixed effect variable, the model showed no significant relation with the number of SNPs under selection (marginal $\mathrm{r}^{2}=0.011$, $\mathrm{p}=0.38$ ). A similar result was obtained when the mean number of individuals was used as the fixed effect variable (Marginal $\mathrm{r}^{2}=0.041, \mathrm{p}=0.16$ ). However, when the number of SNPs was used as the fixed effect variable, a positive and significant relationship was observed with the number of SNP under selection (Fig. 2A). When the total number of individuals was used as a fixed effect variable in the model, a negative and significant relation was observed in relation to the number of SNPs under selection (Fig. 2B). Subsequently, it was verified that the total number of individuals had a negative and significant relation with the number of SNPs used (Fig. 2C).

\section{Discussion}

The data obtained in the systematic review of studies in wild plant populations showed great heterogeneity in the number of individuals, populations, and quantity of SNPs. Our meta-analyses revealed that the total number of individuals and number of SNPs used are of fundamental importance in detecting signs of natural selection in wild plant populations. We also found an enormous knowledge gap in neotropics, underscoring the fact that predicting the response of the tropical forests to geoclimatic changes based on available data is not possible. Most of the studies had been conducted in the biomes of temperate and mixed forests of the European and North American habitats. However, we had to use them to apply the results in the recommendation for other environments, in order to help in decentralizing studies that are performed almost exclusively in the North hemisphere. Thus, it becomes evident the need to increase the possibilities of develop general methodologies guidelines that support decision making about sampling based on what we have available in the literature. Therefore, although our results were mostly based on species from temperate environment, we believe that they can be generalized to global conclusions. For this reason, our discussion focused on tropical regions, as they are recognized as a biodiversity hotspot and having really few studies with landscape genomics.

We found few empirical studies evaluating SNPs under natural selection, with only 36 plants species under analysis. From those studies, we registered ten families, where $53 \%$ of the species belonged to the Pinaceae family. This result evidences the poor understanding of local adaptation processes for different species. Considering that tropical forests have approximately 11,371 species of trees ${ }^{44}$, we have a limited vision to predict how species may respond to anthropogenic changes like climate change, fragmentation, and forest loss ${ }^{45,46}$. In addition, $\sim 81 \%$ of studies were conducted in the European continent or in North America. Although much of the plant diversity is located in tropical regions ${ }^{44,47}$, the ability of these species to adapt in situ in response to environmental changes has not been studied ${ }^{48}$. We believe that this lack of knowledge for the tropics is a reflection of the socioeconomic conditions of this region as well as the lack of specialists in the area since population genomics is relatively recent and the first studies in the tropics are just emerging (see Table 1). Considering that tropical regions could be severely affected by climate change ${ }^{4}$, our results have shown the great need for studies that seek to understand how geoclimatic variables influence local adaptation. Through these studies, it will be possible to predict how tropical species will respond to climate change and assist in their conservation and management ${ }^{29,32,35,45}$.

With our systematic review of empirical studies, we have evidenced that the number of populations and mean number of individuals in a population do not influence the ability to detect natural selection. This emphasizes the importance of sampling, at the best, the whole extent of the area that is being influenced by the environmental variable of interest, rather than focusing on the number of populations sampled or on the mean number of individuals per population. If many populations, from similar environments, are sampled, the probability of detecting local adaptation does not increase ${ }^{39}$. On the other hand, even if few populations are sampled in contrasting environmental conditions, it would increase the power to detect local adaptation ${ }^{39}$. Thus, it will be more effective to cover the environmental heterogeneity, avoiding efforts both in the expansion of the number of populations and in the average number of individuals per population.

The linear mixed-effect model showed that the number of SNPs used explained 59\% of the variation in the number of SNPs under selection and therefore can positively influence the detection of natural selection signals. This could occur because mostly a low percentage of genes in a genome are under natural selection (Table 1); therefore, increasing the genome sampling, also increases the chance of identifying SNPs under selection. Interestingly, a general pattern observed was that studies using Random, Targeted Pool-Seq, and Random Pool-Seq methodologies used more SNPs and had a greater number of SNPs under selection. Therefore, it would be beneficial if future works used one of these methodologies, especially Random Pool-Seq and Targeted Pool-Seq, to optimize the cost-benefit ratio. In this context, studies that aim to evaluate the indicators of natural selection in populations of wild plants could use the Targeted Pool-Seq approach, in cases where genomic information of the species is available (e.g., genome size and gene annotation). By using targeted sequencing in DNA pools, cost benefit ratio of such studies would be increased. In contrast, for the species with little or no genomic information available and for laboratories that are working on landscape genomics for the first time, Random Pool-Seq is the best strategy to increase the chances of detecting SNPs under selection. An interesting alternative 


\begin{tabular}{|c|c|c|c|c|c|c|c|c|}
\hline Author & Species & Pop & Ind & $\begin{array}{l}\text { Ind_- } \\
\text { Pop }\end{array}$ & SNPs & $\begin{array}{l}\text { SNPs } \\
\text { GEA }\end{array}$ & $\begin{array}{l}\text { SNPs } \\
\text { GEA* }\end{array}$ & Method \\
\hline Eckert et al..$^{57}$ & Pinus taeda & 54 & 682 & 12.63 & 1730 & 118 & 6.82 & Targeted \\
\hline Mosca et al..$^{58}$ & Abies alba Mill & 37 & 1183 & 31.97 & 249 & 3 & 1.20 & Targeted \\
\hline Mosca et al..$^{58}$ & Larix decidua Mill & 24 & 920 & 38.33 & 267 & 5 & 1.87 & Targeted \\
\hline Keller et al..$^{59}$ & Populus balsamifera $L$ & 31 & 443 & 14.29 & 335 & 11 & 3.28 & Targeted \\
\hline Prunier et al. ${ }^{60}$ & Picea mariana & 41 & 593 & 14.46 & 47 & 9 & 19.15 & Targeted \\
\hline Mosca et al..$^{58}$ & Pinus cembra $L$ & 24 & 860 & 35.83 & 459 & 11 & 2.40 & Targeted \\
\hline Mosca et al..$^{58}$ & Pinus mugo & 27 & 935 & 34.63 & 693 & 14 & 2.02 & Targeted \\
\hline Fischer et al. $^{23}$ & Arabidopsis halleri & 5 & 100 & 20.00 & 2091957 & 1037 & 0.05 & Random-Pool-Sec \\
\hline Bashalkhanov et al. ${ }^{31}$ & Picea rubens & 5 & 900 & 180.00 & 33 & 6 & 18.18 & Targeted \\
\hline Mosca et al. ${ }^{61}$ & Abies alba Mill & 36 & 1108 & 30.80 & 231 & 5 & 2.16 & Targeted \\
\hline Tsumura et al. ${ }^{62}$ & Cryptomeria japonica & 14 & 186 & 13.29 & 3930 & 25 & 0.64 & Targeted \\
\hline Mosca et al. ${ }^{61}$ & Larix decidua Mill & 22 & 824 & 37.50 & 233 & 7 & 3.00 & Targeted \\
\hline Modesto et al. ${ }^{63}$ & Quercus suber $L$ & 16 & 96 & 6.00 & 44 & 5 & 11.36 & Targeted \\
\hline Scalfi et al. ${ }^{64}$ & Picea abies [L.] Karst) & 12 & 300 & 25.00 & 227 & 2 & 0.88 & Targeted \\
\hline Cullingham et al. $^{46}$ & Pinus contorta var. latifolia & 13 & 368 & 28.31 & 399 & 22 & 5.51 & Targeted \\
\hline Cullingham et al. ${ }^{46}$ & Pinus banksiana & 4 & 100 & 25.00 & 399 & 8 & 2.01 & Targeted \\
\hline Geraldes et al.$^{65}$ & Populus trichocarpa & 25 & 424 & 16.96 & 28135 & 58 & 0.21 & Targeted \\
\hline H De Kort et al. ${ }^{66}$ & Frangula alnus subsp. alnus & 25 & 619 & 24.76 & 183 & 143 & 78.14 & Targeted \\
\hline Hamlin et al. ${ }^{67}$ & Iris hexagona & 8 & 92 & 11.50 & 750 & 70 & 9.33 & Random \\
\hline Eckert et al.$^{68}$ & Pinus lambertiana & 10 & 241 & 24.10 & 475 & 14 & 2.95 & Targeted \\
\hline Jaramillo-Correa et al.$^{69}$ & Pinus pinaster Aiton & 36 & 772 & 21.44 & 266 & 18 & 6.77 & Targeted \\
\hline Roschanski et al..$^{13}$ & Abies alba Mill & 4 & 376 & 94.00 & 267 & 8 & 3.00 & Targeted \\
\hline Christmas et al..$^{25}$ & $\begin{array}{l}\text { Dodonaea viscosa ssp. } \\
\text { Angustissima }\end{array}$ & 17 & 89 & 5.24 & 8462 & 93 & 1.10 & Targeted \\
\hline Pluess et al..$^{10}$ & Fagus sylvatica & 79 & 234 & 2.96 & 144 & 16 & 11.11 & Targeted \\
\hline Gugger et al. ${ }^{30}$ & Quercus lobata & 12 & 22 & 1.83 & 220427 & 79 & 0.04 & Targeted \\
\hline Sork et al. ${ }^{20}$ & Quercus lobata & 13 & 45 & 3.46 & 195 & 8 & 4.10 & Targeted \\
\hline Rellstab et al. ${ }^{12}$ & Quercus robur & 24 & 465 & 19.38 & 3576 & 181 & 5.06 & Targeted-Pool-Sec \\
\hline Rellstab et al. ${ }^{12}$ & Quercus petraea & 18 & 350 & 19.44 & 3576 & 224 & 6.26 & Targeted-Pool-Sec \\
\hline Rellstab et al. ${ }^{12}$ & Quercus pubescens & 17 & 326 & 19.18 & 3576 & 304 & 8.50 & Targeted-Pool-Sec \\
\hline Di Pierro et al. ${ }^{18}$ & Norway spruce & 24 & 826 & 34.40 & 214 & 10 & 4.67 & Targeted \\
\hline Di Pierro et al. ${ }^{18}$ & Picea abies [L.] Karst & 23 & 826 & 35.91 & 214 & 7 & 3.27 & Targeted \\
\hline Mosca et al..$^{19}$ & Pinus cembra $L$ & 18 & 678 & 37.67 & 455 & 74 & 16.26 & Targeted \\
\hline Mosca et al. ${ }^{19}$ & Pinus mugo & 20 & 673 & 33.65 & 663 & 60 & 9.05 & Targeted \\
\hline Rajora $\mathrm{et} \mathrm{al.}{ }^{28}$ & Pinus strobus & 29 & 638 & 22.00 & 44 & 2 & 4.55 & Targeted \\
\hline Di Pierro et al. ${ }^{70}$ & Picea abies [L.] Karst & 18 & 687 & 38.17 & 175 & 19 & 10.86 & Targeted \\
\hline Lind et al. ${ }^{71}$ & Pinus albicaulis Engelm & 8 & 244 & 30.50 & 116231 & 1780 & 1.53 & Random \\
\hline Fahrenkrog et al. ${ }^{72}$ & Populus deltoides & 50 & 168 & 3.36 & 79969 & 2.522 & 3.15 & Targeted-Pool-Sec \\
\hline Frachon et al. ${ }^{54}$ & Arabidopsis thaliana & 168 & 2.574 & 15.32 & 1638649 & 300 & 0.02 & Random-Pool-Sec \\
\hline Lanes et al..$^{73}$ & Ipomoea cavalcantei & 1 & 122 & 122.00 & 34102 & 2239 & 6.57 & Random \\
\hline Lanes et al. ${ }^{73}$ & Ipomoea maurandioides & 4 & 254 & 63.50 & 23181 & 1814 & 7.83 & Random \\
\hline Shih et al. ${ }^{56}$ & $\begin{array}{l}\text { Keteleeria davidiana var. } \\
\text { formosana }\end{array}$ & 5 & 62 & 12.40 & 13914 & 15 & 0.11 & Random-Pool-Sec \\
\hline Martins et al. ${ }^{74}$ & Quercus rugosa Née & 17 & 103 & 6.06 & 5354 & 97 & 1.81 & Random-Pool-Sec \\
\hline Ruiz Daniels et al. ${ }^{75}$ & Pinus halepensis Mill & 46 & 1.326 & 28.83 & 294 & 7 & 2.38 & Targeted \\
\hline Alam et al. ${ }^{76}$ & $\begin{array}{l}\text { Vaccinium vitis-idaea } \\
\text { subsp. Minus }\end{array}$ & 56 & 56 & 1.00 & 1586 & 132 & 8.32 & Random \\
\hline \multirow{4}{*}{ Descriptive statistics } & Minimum & 1 & 22 & 1.00 & 33 & 2 & 0.02 & \\
\hline & Maximum & 168 & 2574 & 180 & 2091957 & 2522 & 78.14 & \\
\hline & Mean & 25.91 & 520.2 & 29.48 & 97420 & 263.2 & 7 & \\
\hline & Standard deviation & 27.28 & 471.82 & 32.31 & 395181.4 & 614 & 11.99 & \\
\hline
\end{tabular}

Table 1. Biological models and descriptive statistics calculated in 35 studies that assessed local adaptation in wild plant populations in situ using markers SNPs. (Ind, Number of individuals; Ind_Pop, Average number of individuals per population; SNPs, Number of used SNPs; Pop, Number of populations; SNPs_GEA, Number of SNPs under selection; SNPs_GEA*, Percentage of SNPs under selection; Method, Method used to generate the SNP data). 

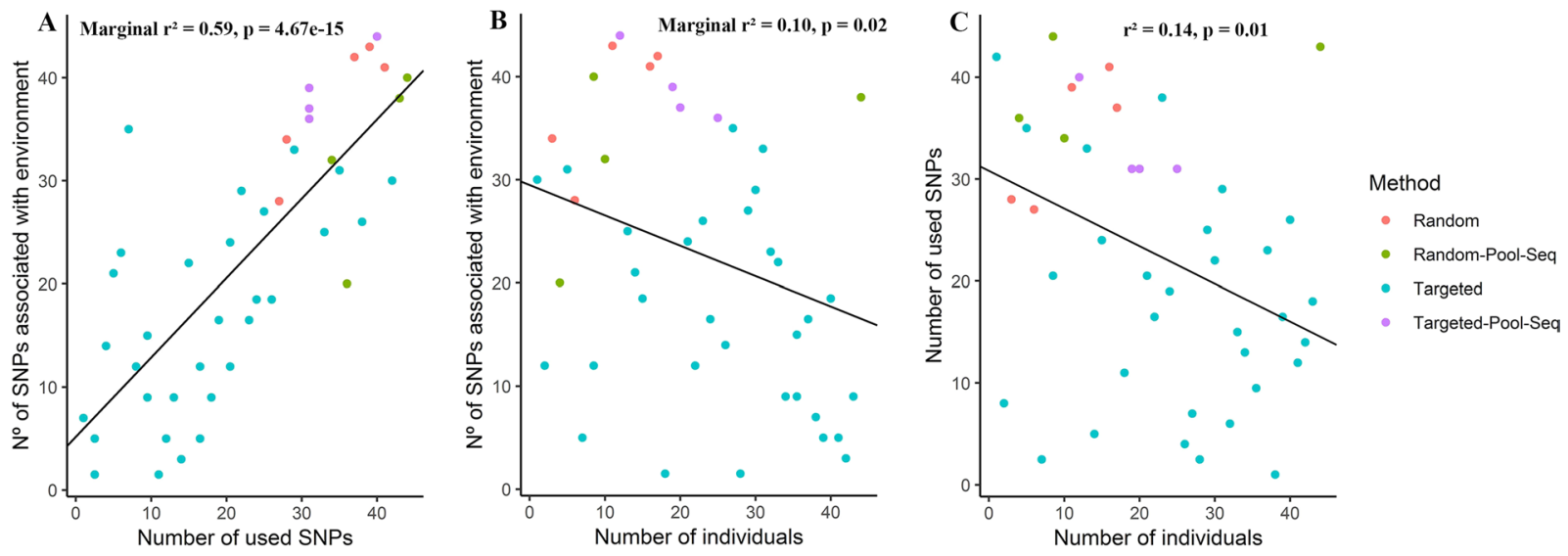

Figure 2. Relation between sampling design and the detection of SNPs with environmental association (A) number of SNPs with environmental association as a function of the number of SNPs used in the studies; (B) number of SNPs with environmental association as a function of the total number of individuals in each study; (C) number of SNPs used as a function of the total number of individuals in each study. The colors of the dots represent the different methods used in the studies (Random used random regions of DNA and individualized sequencing; Random Pool-Seq used random regions of DNA and Pool-Seq technique; Targeted used only gene regions or expressed sequence tags and individualized sequencing; Targeted Pool-Seq used only gene regions or expressed sequence tags and sequencing in Pool-Seq).

to make landscape genomics financially feasible would be the use of grouped sequencing of individuals by techniques like RADseq that do not require any prior knowledge of the genome ${ }^{49}$. While it is still advantageous to use the Pool-Seq technique which can increase accuracy in allele frequency estimates, compared to individualized sequencing, making it an excellent tool to be used in landscape genomic studies ${ }^{50-52}$. Moreover, it also allows to reconcile the sampling of a large number of individuals needed in population genomics, with thousands or millions of SNPs, to evaluate adaptation in natural populations ${ }^{12,23,53,54}$. It is important to note that the Pool-Seq technique has some limitations, for example, not providing information for each individual separately ${ }^{52}$. Therefore, the use of this technique is only suitable for studies aiming for population inferences without the need of information about individuals.

By fixing the variable in the linear mixed effects model as total number of individuals, we found that the total sample size was inversely related to the number of SNPs under selection and to the number of SNPs used. Although significant, these correlations explain only $10 \%$ of the variation in the number of SNPs under selection and $14 \%$ in the number of SNPs used. However, much of the variation in the number of SNPs under selection (59\%) could be explained by fixing the variable as the number of SNPs used. We therefore, believe that the significantly negative correlation between the total number of individuals and the number of SNPs under selection is a reflection of the inverse relationship observed between the total number of individuals and the number of SNPs used. Thus, the negative relation seen between the total number of individuals and the number of SNPs under selection is an artifact generated by the lower capacity detection of natural selection when the number of SNPs used decreases. The negative relationship observed between the number of individuals sampled and the number of SNPs used could be due to incomplete genomic information because of the costs associated with sequencing of whole genomes ${ }^{53}$. Among species for which some genomic information is available, size consideration of the genome becomes important; because the larger genomes may need more number of SNPs to be sampled to give a full perspective, thus impacting the number of individuals that can be analyzed given the budgetary constraints. Also, investing a huge portion of the budget for sampling in the field to increase the sample size, would affect the laboratory stages of the study by limiting resource allocation. Thus, the negative relation found in this study, can be related to the costs of individual sequencing of each sample. In population genomics studies, the conflicting demands between the total number of individuals and the number of SNPs used is circumvented by using the Pool-Seq technique ${ }^{53}$ (Table 1). This technique has also been used in plant species with incomplete or total absence of genomic information ${ }^{52}$. This indicates that the same strategy can be used in the landscape genomics studies of species in tropical regions, which are major biodiversity hotspots of the world ${ }^{44,47}$ with little or no genomic information available.

\section{Guidance for Study of Local Adaptation in Wild Plant Species}

Our study involving systematic review of empirical studies and meta-analysis of data and results therein, about wild plant populations, allows us to put forth the recommendations that in future landscape genomics studies, the experimental design should focus on increasing the total number of individuals sampled along the environmental heterogeneity under analysis ${ }^{39}$. In addition, we also suggest that researchers seek to evaluate the adaptation in wild plant populations using the Pool-Seq technique to increase the number of SNPs and accuracy in allele frequency estimates ${ }^{52}$. This technique has been used in population genomics as a powerful tool under different study scenarios, including model species ${ }^{54}$, allopolyploid species ${ }^{55}$ as well as for species with little or no genomic information available ${ }^{56}$. Following these guidelines, it will be possible to extrapolate findings from studies that 
are performed almost exclusively on species of temperate climate and expanding them to tropical species. In this way, landscape genomics can be conducted in tropical regions in relation to anthropogenic changes, in spite of existing budgetary constraints, and the data so generated can be used to develop strategies for management and conservation of biodiversity.

Received: 8 May 2019; Accepted: 11 February 2020;

Published online: 28 February 2020

\section{References}

1. Liu, B., Su, J., Chen, J., Cui, G. \& Ma, J. Anthropogenic Halo Disturbances Alter Landscape And Plant Richness: A Ripple Effect. PLoS One 8, 1-8 (2013).

2. Wilson, M. C. et al. Habitat Fragmentation And Biodiversity Conservation: Key Findings And Future Challenges. Landsc. Ecol. 31, 219-227 (2016).

3. Fahrig, L. Effects Of Habitat Fragmentation On Biodiversity. Annu. Rev. Ecol. Evol. Syst. 34, 487-515 (2003).

4. Bellard, C., Bertelsmeier, C., Leadley, P., Thuiller, W. \& Courchamp, F. Impacts Of Climate Change On The Future Of Biodiversity. Ecol. Lett. 15, 365-377 (2012)

5. Parmesan, C. \& Hanley, M. E. Plants And Climate Change: Complexities And Surprises. Ann. Bot. 116, 849-864 (2015)

6. Davis, M. B., Shaw, R. G. \& Etterson, J. R. Evolutionary Responses To Changing Climate. Ecology 86, 1704-1714 (2005)

7. Nicotra, A. B. et al. Plant Phenotypic Plasticity In A Changing Climate. Trends Plant Sci. 15, 684-692 (2010).

8. Alberto, F. J. et al. Potential For Evolutionary Responses To Climate Change - Evidence From Tree Populations. Glob. Chang. Biol., https://doi.org/10.1111/gcb.12181 (2013).

9. Kawecki, T. J. \& Ebert, D. Conceptual Issues In Local Adaptation. Ecol. Lett. 7, 1225-1241 (2004).

10. Pluess, A. R. et al. Genome - Environment Association Study Suggests Local Adaptation To Climate At The Regional Scale In Fagus Sylvatica. New Phytol. 210, 589-601 (2016).

11. Rellstab, C. et al. Local Adaptation (Mostly) Remains Local: Reassessing Environmental Associations Of Climate-Related Candidate Snps In Arabidopsis Halleri. Heredity (Edinb). 118, 193-201 (2017).

12. Rellstab, C. et al. Signatures Of Local Adaptation In Candidate Genes Of Oaks (Quercus Spp.) With Respect To Present And Future Climatic Conditions. Mol. Ecol. 25, 5907-5924 (2016).

13. Roschanski, A. M. et al. Evidence Of Divergent Selection For Drought And Cold Tolerance At Landscape And Local Scales In Abies Alba Mill. In The French Mediterranean Alps. Mol. Ecol. 25, 776-794 (2016).

14. Steane, D. A. et al. Genome-Wide Scans Detect Adaptation To Aridity In A Widespread Forest Tree Species. Mol. Ecol. 23, 2500-2513 (2014).

15. Rellstab, C., Gugerli, F., Eckert, A. J., Hancock, A. M. \& Holderegger, R. A Practical Guide To Environmental Association Analysis In Landscape Genomics. Mol. Ecol. 24, 4348-4370 (2015).

16. Manel, S. et al. Perspectives On The Use Of Landscape Genetics To Detect Genetic Adaptive Variation In The Field. Mol. Ecol. 19, 3760-3772 (2010).

17. Turner, T. L., Bourne, E. C., Von Wettberg, E. J., Hu, T. T. \& Nuzhdin, S. V. Population Resequencing Reveals Local Adaptation Of Arabidopsis Lyrata To Serpentine Soils. Nat. Genet. 42, 260-263 (2010).

18. Pierro, E. A. D. et al. Climate-Related Adaptive Genetic Variation And Population Structure In Natural Stands Of Norway Spruce In The South-Eastern Alps. Tree Genet. Genomes 12, 16, https://doi.org/10.1007/s11295-016-0972-4 (2016).

19. Mosca, E., Gugerli, F., Eckert, A. J. \& Neale, D. B. Signatures Of Natural Selection On Pinus Cembra And P. Mugo Along Elevational Gradients In The Alps. Tree Genet. Genomes, https://doi.org/10.1007/s11295-015-0964-9 (2016).

20. Sork, V. L. et al. Landscape Genomic Analysis Of Candidate Genes For Climate Adaptation In A California Endemic Oak, Quercus Lobata. Am. J. Bot. 103, 33-46 (2016).

21. Ćalić, I., Bussotti, F., Martínez-García, P. J. \& Neale, D. B. Recent Landscape Genomics Studies In Forest Trees - What Can We Believe? Tree Genet. Genomes 12, 3 (2016).

22. Gaut, B. Arabidopsis Thaliana As A Model For The Genetics Of Local Adaptation. Nat. Genet. 44, 732-732 (2012).

23. Fischer, M. C. et al. Population Genomic Footprints Of Selection And Associations With Climate In Natural Populations Of Arabidopsis Halleri From The Alps. Mol. Ecol. 22, 5594-5607 (2013).

24. Andrews, K. R. \& Luikart, G. Recent Novel Approaches For Population Genomics Data Analysis. Mol. Ecol. 23, 1661-1667 (2014).

25. Christmas, M. J., Biffin, E., Breed, M. F. \& Lowe, A. J. Finding Needles In A Genomic Haystack: Targeted Capture Identifies Clear Signatures Of Selection In A Nonmodel Plant Species. Mol. Ecol. 25, 4216-4233 (2016).

26. Parisod, C. \& Holderegger, R. Adaptive Landscape Genetics: Pitfalls And Benefits Adaptive Landscape Genetics: Pitfalls. Mol. Biol. Evol. 21, 3644-3646 (2012).

27. Zhou, Y., Zhang, L., Liu, J., Wu, G. \& Savolainen, O. Climatic Adaptation And Ecological Divergence Between Two Closely Related Pine Species In Southeast China. Mol. Ecol. 23, 3504-3522 (2014).

28. Rajora, O. P., Eckert, A. J. \& Zinck, J. W. R. Single-Locus Versus Multilocus Patterns Of Local Adaptation To Climate In Eastern White Pine (Pinus Strobus, Pinaceae). PLoS One 11, 1-26 (2016).

29. Fitzpatrick, M. C. \& Keller, S. Ecological Genomics Meets Community-Level Modelling Of Biodiversity: Mapping The Genomic Landscape Of Current And Future Environmental. Ecol. Lett. 18, 1-16 (2015).

30. Gugger, P. F., Cokus, S. J. \& Sork, V. L. Association Of Transcriptome-Wide Sequence Variation With Climate Gradients In Valley Oak (Quercus Lobata). Tree Genet. Genomes 12, 1-14 (2016).

31. Bashalkhanov, S., Eckert, A. J. \& Rajora, O. P. Genetic Signatures Of Natural Selection In Response To Air Pollution In Red Spruce (Picea Rubens, Pinaceae). Mol. Ecol. 22, 5877-5889 (2013).

32. Shafer, A. B. et al. Genomics And The Challenging Translation Into Conservation Practice. Trends Ecol. Evol. 30, $78-87$ (2015).

33. Schoville, S. D. et al. Adaptive Genetic Variation On The Landscape: Methods And Cases Adaptive Genetic Variation On The Landscape: Methods And Cases. Annu. Rev. Ecol. Evol. Syst. 43, 23-43 (2012).

34. Robasky, K., Lewis, N. E. \& Church, G. M. The Role Of Replicates For Error Mitigation In Next-Generation Sequencing. Nat. Rev. Genet. 15, 56 (2014).

35. Benestan, L. M. et al. Conservation Genomics Of Natural And Managed Populations: Building A Conceptual And Practical Framework. Mol. Ecol. 25, 2967-2977 (2016).

36. Manel, S. et al. Genomic Resources And Their Influence On The Detection Of The Signal Of Positive Selection In Genome Scans. Mol. Ecol. 25, 170-184 (2016).

37. Ahrens, C. W. et al. The Search For Loci Under Selection: Trends, Biases And Progress. Mol. Ecol. https://doi.org/10.1111/mec.14549 (2018).

38. Mita, S. D. E. et al. Detecting Selection Along Environmental Gradients: Analysis Of Eight Methods And Their Effectiveness For Outbreeding And Selfing Populations. Mol. Ecol. 22, 1383-1399 (2013).

39. Lotterhos, K. E. \& Whitlock, M. C. The Relative Power Of Genome Scans To Detect Local Adaptation Depends On Sampling Design And Statistical Method. Mol. Ecol. 24, 1031-1046 (2015). 
40. Forester, B. R., Jones, M. R., Joost, S., Landguth, E. L. \& Lasky, J. R. Detecting Selection In Natural Populations: Making Sense Of Detecting Spatial Genetic Signatures Of Local Adaptation In Heterogeneous Landscapes. Mol. Ecol. 25, 104-120 (2016).

41. Bates, D., Mächler, M., Bolker, B. M. \& Walker, S. C. Fitting Linear Mixed-Effects Models Using Lme4. J. Stat. Softw. Oct. 67 (2015).

42. Nakagawa, S. \& Schielzeth, H. A General And Simple Method For Obtaining R 2 From Generalized Linear Mixed-Effects Models. Methods Ecol. and Evolution 4, 133-142 (2013).

43. H, W. ggplot2: Elegant Graphics For Data Analysis. Springer (2016).

44. Slik, J. W. F. et al. An Estimate Of The Number Of Tropical Tree Species. Pnas 112, E4628-E4629 (2015).

45. Hansen, M. M., Olivieri, I., Waller, D. M. \& Nielsen, E. E. Monitoring Adaptive Genetic Responses To Environmental Change. Mol. Ecol. 21, 1311-1329 (2012).

46. Cullingham, C. I., Cooke, J. E. K. \& Coltman, D. W. Cross-Species Outlier Detection Reveals Different Evolutionary Pressures Between Sister Species. New Phytol. 204, 215-229 (2014).

47. Mannion, P. D., Upchurch, P., Benson, R. B. J. \& Goswami, A. The Latitudinal Biodiversity Gradient Through Deep Time. Trends Ecol. Evol. 29, 42-50 (2014).

48. Storfer, A., Murphy, M. A., Spear, S. F., Holderegger, R. \& Waits, L. P. Landscape Genetics: Where Are We Now? Mol. Ecol. 19, 3496-3514 (2010).

49. Andrews, K. R., Good, J. M., Miller, M. R., Luikart, G. \& Hohenlohe, P. A. Harnessing The Power Of Radseq For Ecological And Evolutionary Genomics. Nat. Rev. Genet. 17, 81-92 (2016).

50. Futschik, A. \& Schlötterer, C. The Next Generation Of Molecular Markers From Massively Parallel Sequencing Of Pooled DNA Samples. Genetics 186, 207-218 (2010).

51. Bansal, V., Tewhey, R., Leproust, E. M. \& Schork, N. J. Efficient And Cost Effective Population Resequencing By Pooling And InSolution Hybridization. PLoS One 6, 1-6 (2011).

52. Schlötterer, C., Tobler, R., Kofler, R. \& Nolte, V. Sequencing Pools Of Individuals - Mining Genome-Wide Polymorphism Data Without Big Funding. Nat. Rev. Genet. 15, 749 (2014).

53. Gautier, M. et al. Estimation Of Population Allele Frequencies From Next-Generation Sequencing Data: Pool-Versus IndividualBased Genotyping. Mol. Ecol. 22, 3766-3779 (2013).

54. Frachon, L. et al. A Genomic Map Of Climate Adaptation In Arabidopsis Thaliana At A Micro-Geographic Scale. Front. Plant Sci. 9 , $1-15$ (2018).

55. Hirao, A. S. et al. Cost-Effective Discovery Of Nucleotide Polymorphisms In Populations Of An Allopolyploid Species Using PoolSeq. Am. J. Mol. Biol. 7, 153-168 (2017).

56. Shih, K., Chang, C., Chung, J., Chiang, Y. \& Hwang, S.-Y. Adaptive Genetic Divergence Despite Significant Isolation-By-Distance In Populations Of Taiwan Cow-Tail Fir (Keteleeria Davidiana Var. Formosana). Front. Plant Sci. 9 (2018).

57. Eckert, A. J. et al. Back To Nature: Ecological Genomics Of Loblolly Pine (Pinus Taeda, Pinaceae). Mol. Ecol. 19, 3789-3805 (2010).

58. Mosca, E. et al. The Geographical And Environmental Determinants Of Genetic Diversity For Four Alpine Conifers Of The European Alps. Mol. Ecol. 21, 5530-5545 (2012).

59. Keller, S. R., Levsen, N., Olson, M. S. \& Tiffin, P. Local Adaptation in the Flowering-Time Gene Network of Balsam Poplar, Populus balsamifera L. Mol. Biol. Evol. 29, 3143-3152 (2012).

60. Prunier, J., GÉrardi, S., Laroche, J., Beaulieu, J. \& Bousquet, J. Parallel And Lineage-Specific Molecular Adaptation To Climate In Boreal Black Spruce. Mol. Ecol. 21, 4270-4286 (2012).

61. Mosca, E., González-Martínez, S. C. \& Neale, D. B. Environmental Versus Geographical Determinants Of Genetic Structure In Two Subalpine Conifers. New Phytol. 201, 180-192 (2014).

62. Tsumura, Y. et al. Genetic Differentiation And Evolutionary Adaptation In Cryptomeria Japonica. G3 Genes|Genomes|Genetics 4, 2389-402 (2014).

63. Modesto, I. S. et al. Identifying Signatures Of Natural Selection In Cork Oak (Quercus Suber L.) Genes Through SNP Analysis. Tree Genet. Genomes 10, 1645-1660 (2014).

64. Scalfi, M. et al. Micro- And Macro-Geographic Scale Effect On The Molecular Imprint Of Selection And Adaptation In Norway Spruce. PLoS Genet. 9, 1-22 (2014).

65. Geraldes, A. et al. Landscape Genomics Of Populus Trichocarpa: The Role Of Hybridization, Limited Gene Flow, And Natural Selection In Shaping Patterns Of Population Structure. Evolution (N. Y). 68, 3260-3280 (2014).

66. Kort, H. D., Vandepitte, K., Mergeay, J., Mijnsbrugge, K. V. \& Honnay, O. The Population Genomic Signature Of Environmental Selection In The Widespread Insect-Pollinated Tree Species Frangula Alnus At Different Geographical Scales. Heredity (Edinb). 115, $415-425$ (2015).

67. Hamlin, J. A. P. \& Arnold, M. L. Neutral And Selective Processes Drive Population Differentiation For Iris Hexagona. J. Hered. 628-636, https://doi.org/10.5061/dryad.4n15b (2015).

68. Eckert, A. J. et al. Local Adaptation At Fine Spatial Scales: An Example From Sugar Pine (Pinus Lambertiana, Pinaceae). Tree Genet. Genomes 11 (2015).

69. Jaramillo-Correa, J.-P. et al. Molecular Proxies For Climate Maladaptation In A Long-Lived Tree (Pinus Pinaster Aiton, Pinaceae). Genetics 199, 793-807 (2015).

70. Pierro, E. A. D. et al. Adaptive Variation In Natural Alpine Populations Of Norway Spruce (Picea Abies [L.] Karst) At Regional Scale: Landscape Features And Altitudinal Gradient Effects. For. Ecol. Manage. 405, 350-359 (2017).

71. Lind, B. M. et al. Water Availability Drives Signatures Of Local Adaptation In Whitebark Pine (Pinus Albicaulis Engelm.) Across Fine Spatial Scales Of The Lake Tahoe Basin, USA. Mol. Ecol. 26, 3168-3185 (2017).

72. Fahrenkrog, A. M. et al. Population Genomics Of The Eastern Cottonwood (Populus Deltoides). Ecol. Evol. 9426-9440, https://doi. org/10.1002/ece3.3466 (2017)

73. Lanes, É. C. et al. Landscape Genomic Conservation Assessment Of A Narrow-Endemic And A Widespread Morning Glory From Amazonian Savannas. Front. Genet. 9, 1-13 (2018).

74. Martins, K. et al. Landscape Genomics Provides Evidence Of Climate-Associated Genetic Variation In Mexican Populations Of Quercus Rugosa. Evol. Appl. 11, 1842-1858 (2018).

75. Daniels, R. R. et al. Inferring Selection In Instances Of Long - Range Colonization: The Aleppo Pine (Pinus Halepensis) In The Mediterranean Basin. Mol. Ecol. 27, 3331-3345 (2018).

76. Alam, Z., Roncal, J. \& Peña-Castillo, L. Genetic Variation Associated With Healthy Traits And Environmental Conditions In Vaccinium Vitis-Idaea. BMC Genomics 19, 1-13 (2018).

\section{Acknowledgements}

The authors thank the Brazilian National Council of Scientific and Technological Development (CNPq) for the FAG research productivity fellowship; and Coordination for the Improvement of Higher Education Personnel (CAPES) for granting a scholarship to ASS. The authors also thank Marina Corrêa Côrtes, Luciana Aparecida Carlini Garcia, and Ricardo Dobrovolski for the corrections in the first version of the manuscript. The authors acknowledge Dr. Leandro L. Loguercio and Dra. Thâmara M. Lima who also helped on the writing of the resubmitting letter. 


\section{Author contributions}

A.S.S. and F.A.G. contributed to the design, analysis and writing of the manuscript.

\section{Competing interests}

The authors declare no competing interests.

\section{Additional information}

Correspondence and requests for materials should be addressed to A.S.S.

Reprints and permissions information is available at www.nature.com/reprints.

Publisher's note Springer Nature remains neutral with regard to jurisdictional claims in published maps and institutional affiliations.

(c) (i) Open Access This article is licensed under a Creative Commons Attribution 4.0 International License, which permits use, sharing, adaptation, distribution and reproduction in any medium or format, as long as you give appropriate credit to the original author(s) and the source, provide a link to the Creative Commons license, and indicate if changes were made. The images or other third party material in this article are included in the article's Creative Commons license, unless indicated otherwise in a credit line to the material. If material is not included in the article's Creative Commons license and your intended use is not permitted by statutory regulation or exceeds the permitted use, you will need to obtain permission directly from the copyright holder. To view a copy of this license, visit http://creativecommons.org/licenses/by/4.0/.

(C) The Author(s) 2020 